\title{
Effect of Winkler foundation, inhomogeneity and orthotropy on the frequency of plates
}

\author{
V.C. Haciyev*, G.R. Mirzeyeva and A.I. Shiriyev \\ National Academy of Sciences of Azerbaijan, Institute of Mathematics and Mechanics, Baku, Azerbaijan
}

\begin{abstract}
This study investigates the frequencies of inhomogeneous orthotropic plates on elastic foundation. The inhomogeneity of orthotropic materials varies linearly with the coordinates of length and thickness. The basic equation is derived applying the Donnell-Mushtari theory and solved using the Galerkin method. The effects of inhomogeneity, Winkler elastic foundation and orthotropy on the frequencies are investigated in detail.
\end{abstract}

\section{Keywords}

Free vibration; Inhomogeneous orthotropic materials; Plates; Winkler elastic foundation

Received: 14 March 2018; Accepted: 21 March 2018

ISSN: 2630-5763 (online) @ 2018 Golden Light Publishing® All rights reserved.

\section{Introduction}

Inhomogeneous structures are widely used in aviation, aerodynamic structure, space vehicles, light-alloy structure of cars and in other engineering structures. The increase in the number of constructive variables extends the possibilities of advanced composite materials, as well as stability and vibration behaviors may be significantly altered. The reason for the appearance of heterogeneity of the material can be manufacturing technology, thermal and mechanical treatment, heterogeneity of compositions and a number of other reasons [1]. Significant contributions to consider different types of inhomogeneity are given in references [2-7]. In many practical applications, composite plates are in contact with soils or other solid particles and can have significant and unavoidable effect on their behaviors. A comprehensive review of elastic foundation models is discussed in the work by Gorbunov-Posadov et al. [8]. Vibration of homogeneous and nonhomogeneous orthotropic plates resting on elastic foundation, which has practical applications in civil, mechanical, marine and aerospace engineers have been extensively studied by using various analytical and numerical methods [9-15].

In majority of the above-mentioned-studies, the elastic properties of inhomogeneous orthotropic materials were varied depending on the coordinate of the thickness or the coordinates in the plane, separately. The proposed study describes the free vibration of heterogeneous orthotropic plates on the Winkler elastic foundation, in which the inhomogeneity linearly varies along the length and thickness together.

\section{Formulation of the problem}

The configuration of rectangular inhomogeneous orthotropic plate with the length $a$, the width $b$ and the thickness $h$ resting on Winkler elastic

\footnotetext{
* Corresponding author

E-mail: vagif.haciyev.imm@gmail.com
} 
foundation is illustrated in Fig. 1. The plate referred to a system of rectangular coordinate system Oxyz. The mid-plane being $z=0$ and the origin is at one corners of the orthotropic plate. $z=0$. The $x$ and $y$ axes are taken along the principle directions of orthotropy and $z$ axis is normal to them.

It is assumed that Young's moduli and shear modulus of the heterogeneous orthotropic plate vary linearly with the coordinates $x$ and $z$ as

$$
\begin{aligned}
& E_{1}=E_{1}^{0}\left[1+\frac{x}{a}\left(\mu_{1}-1\right)\right]\left[1+\mu_{2} \frac{z}{h}\right] \\
& E_{2}=E_{2}^{0}\left[1+\frac{x}{a}\left(\mu_{1}-1\right)\right]\left[1+\mu_{2} \frac{z}{h}\right] \\
& G_{12}=G_{12}^{0}\left[1+\frac{x}{a}\left(\mu_{1}-1\right)\right]\left[1+\mu_{2} \frac{z}{h}\right]
\end{aligned}
$$

while density $\rho$ and Poisson's ratios $v_{1}$ and $v_{2}$ are assumed to be constant [2-7]. Here, $E_{1}^{0}$ and $E_{2}^{0}$ are the Young's moduli in $x$ and $y$ directions, $G_{12}^{0}$ is the shear modulus and $\rho$ is the density of the homogeneous orthotropic plate. Here $X=x / a$ and $Z=z / h$ are the dimensionless variables in $x$ and $z$ directions; $\mu_{1}$ and $\mu_{2}$ are non-homogeneity parameters varied from zero to unity. As $\mu_{1}=1$ and $\mu_{2}=0$, the heterogeneous material is converted into a homogeneous material.

\section{Basic relations}

Based on the classical shell theory, the relationships between the stresses and strains at an arbitrary point of inhomogeneous orthotropic plates are written in the following form [2-7]:

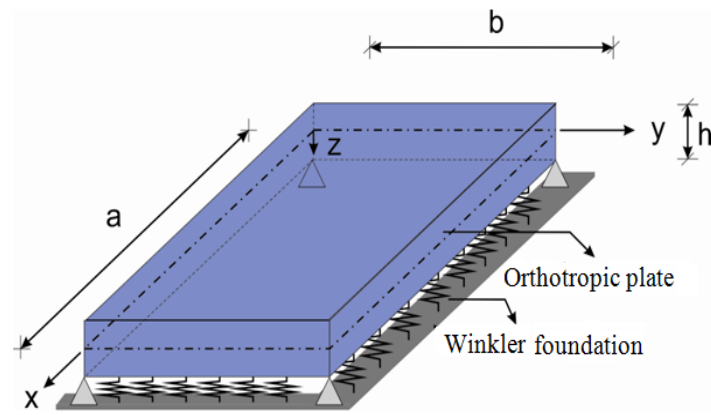

Fig. 1. The inhomogeneous orthotropic rectangular plate on the elastic foundation and the coordinate system

$$
\begin{aligned}
\sigma_{11}= & \frac{1}{1-v_{1} v_{2}} E_{1}^{0}\left[1+\frac{x}{a}\left(\mu_{1}-1\right)\right]\left[1+\mu_{2} \frac{z}{h}\right] \\
& \times\left[e_{11}+v_{1} e_{22}-z\left(\frac{\partial^{2} w}{\partial x^{2}}+v_{1} \frac{\partial^{2} w}{\partial y^{2}}\right)\right] \\
\sigma_{22} & =\frac{1}{1-v_{1} v_{2}} E_{2}^{0}\left[1+\frac{x}{a}\left(\mu_{1}-1\right)\right]\left[1+\mu_{2} \frac{z}{h}\right] \\
& \times\left[e_{22}+v_{2} e_{11}-z\left(\frac{\partial^{2} w}{\partial y^{2}}+v_{2} \frac{\partial^{2} w}{\partial x^{2}}\right)\right] \\
\sigma_{12}= & G_{12}^{0}\left[1+\frac{x}{a}\left(\mu_{1}-1\right)\right]\left[1+\mu_{2} \frac{z}{h}\right] \\
& \times\left(e_{12}-2 z \frac{\partial^{2} w}{\partial x \partial y}\right)
\end{aligned}
$$

where $w$ is the small displacement in $z$ direction and $e_{i j}(i, j=1,2)$ are the strains in the middle plane of inhomogeneous orthotropic plate.

The force and moment resultants are expressed by the following relations $[15,16]$ :

$$
\begin{aligned}
& \left(T_{11}, T_{22}, T_{12}, M_{11}, M_{22}, M_{12}\right) \\
& \quad=\int_{-h / 2}^{h / 2}\left(\sigma_{11}, \sigma_{22}, \sigma_{12}, z \sigma_{11}, z \sigma_{22}, z \sigma_{12}\right) d z
\end{aligned}
$$

Since there are no external forces in the plane of plate $\left(T_{11}=T_{22}=T_{12}=0\right)$, it is therefore assumed that the resultant forces are everywhere is equal to zero. In this case, the following conditions can be written:

$$
\begin{aligned}
& A_{1}\left(e_{11}+v_{1} e_{22}\right)-A_{2}\left(\chi_{11}+v_{1} \chi_{22}\right)=0 \\
& A_{1}\left(e_{22}+v_{2} e_{11}\right)-A_{2}\left(\chi_{22}+v_{2} \chi_{11}\right)=0 \\
& A_{1} e_{12}-A_{2} \chi_{12}=0
\end{aligned}
$$

where $A_{1}=h, A_{2}=\mu_{2} h^{2} / 12$ and $\chi_{i j}(i, j=1,2)$ are the curvatures of the deformed plate.

Taking into account Eq. (2) in the expression (3), after integrating, we obtain the following expressions for the moments:

$$
\begin{aligned}
& M_{11}=-\left(1-\frac{\mu_{2}^{2}}{12}\right) D_{1}^{0}\left[1+\frac{x}{a}\left(\mu_{1}-1\right)\right]\left(\frac{\partial^{2} w}{\partial x^{2}}+v_{1} \frac{\partial^{2} w}{\partial y^{2}}\right) \\
& M_{22}=-\left(1-\frac{\mu_{2}^{2}}{12}\right) D_{2}^{0}\left[1+\frac{x}{a}\left(\mu_{1}-1\right)\right]\left(\frac{\partial^{2} w}{\partial y^{2}}+v_{2} \frac{\partial^{2} w}{\partial x^{2}}\right) \\
& M_{12}=-\left(1-\frac{\mu_{2}^{2}}{12}\right) D_{12}^{0}\left[1+\frac{x}{a}\left(\mu_{1}-1\right)\right] \frac{\partial^{2} w}{\partial x \partial y}
\end{aligned}
$$


where $D_{1}^{0}, D_{2}^{0}, D_{12}^{0}$ are flexural rigidities of the homogeneous orthotropic plate and are defined as:

$$
D_{1}^{0}=\frac{E_{1}^{0} h^{3}}{12\left(1-v_{1} v_{2}\right)}, D_{2}^{0}=\frac{E_{2}^{0} h^{3}}{12\left(1-v_{1} v_{2}\right)}, D_{12}^{0}=\frac{G_{12}^{0} h^{3}}{12}
$$

The partial differential equation of the motion of orthotropic plate resting on the Winkler elastic foundation can be written as [15]:

$\frac{\partial^{2} M_{11}}{\partial x^{2}}+2 \frac{\partial^{2} M_{12}}{\partial x \partial y}+\frac{\partial^{2} M_{22}}{\partial y^{2}}-K w-\rho h \frac{\partial^{2} w}{\partial t^{2}}=0$

where $K$ (in $N / \mathrm{m}^{3}$ ) is the modulus of subgrade reaction for the Winkler elastic foundation $[15,16]$.

Substituting Eq. (5) into Eq. (7), after elementary transformations, we obtain the equation of motion of inhomogeneous orthotropic plate resting on the Winkler elastic foundation as:

$$
\begin{aligned}
& {\left[1+\frac{x}{a}\left(\mu_{1}-1\right)\right] \times\left[D_{1}^{0} \frac{\partial^{4} w}{\partial x^{4}}+D_{2}^{0} \frac{\partial^{4} w}{\partial y^{4}}\right.} \\
& \left.+\left(D_{1}^{0} v_{2}+v_{1} D_{2}^{0}+4 D_{12}^{0}\right) \frac{\partial^{4} w}{\partial x^{2} \partial y^{2}}\right] \\
& +2 D_{1}^{0} \frac{\mu_{1}-1}{a}\left(\frac{\partial^{3} w}{\partial x^{3}}+v_{1} \frac{\partial^{3} w}{\partial y^{2} \partial x}\right)+4 D_{12}^{0} \frac{\mu_{1}-1}{a} \frac{\partial^{3} w}{\partial x \partial y^{2}} \\
& +\frac{12}{12-\mu_{2}^{2}} K w+\frac{12}{12-\mu_{2}^{2}} \rho h \frac{\partial^{2} w}{\partial t^{2}}=0
\end{aligned}
$$

\section{The solution of equation of motion}

We assume that the boundary conditions for the bending of continuous inhomogeneous orthotropic plates coincide with the usual ones in the homogeneous isotropic plate.

We take the harmonic solution of Eq. (8) in the form $[15,16]$

$$
w(x, y, t)=A \sin \frac{m \pi x}{a} \sin \frac{n \pi y}{b} e^{i \omega t}
$$

which satisfies the simply-supported boundary conditions edges of the inhomogeneous orthotropic rectangular plates, here $i=\sqrt{-1}, m$ and $n$ are wave numbers, and $A$ is the unknown amplitude.

Substituting Eq. (9) into Eq. (8), then applying the Galerkin's method to the resulting equation for $m=n=1$, the expression for the frequency of free vibration of inhomogeneous orthotropic plates resting on the Winkler elastic foundation is obtained as:

$$
\begin{aligned}
\omega & =\left(\frac{K_{w}}{\rho h}+\frac{\left(12-\mu_{2}^{2}\right)}{12} \frac{\left(1+\mu_{1}\right)}{2 \rho h}\right. \\
& \left.\times\left[\eta_{1}^{4} D_{1}^{0}+\eta_{2}^{4} D_{2}^{0}+\eta_{1}^{2} \eta_{2}^{2}\left(D_{1}^{0} v_{2}+D_{2}^{0} v_{1}+4 D_{12}^{0}\right)\right]\right)^{1 / 2}
\end{aligned}
$$

where $\eta_{1}=\pi / a$ and $\eta_{2}=\pi / b$.

The values of the dimensionless frequency parameter for inhomogeneous orthotropic plates resting on the Winkler elastic foundation is found as:

$$
\omega_{1}=\omega \frac{a^{2}}{h} \sqrt{\rho / E_{2}^{0}}
$$

As $\mu_{1}=1, \mu_{2}=0$ and $K_{w}=0$, the frequency coincides with the frequency of the homogeneous orthotropic plate without an elastic foundation and can be expressed as:

$$
\omega_{0}=\sqrt{\frac{\eta_{1}^{4} D_{1}^{0}+\eta_{2}^{4} D_{2}^{0}+\eta_{1}^{2} \eta_{2}^{2}\left(D_{1}^{0} v_{2}+D_{2}^{0} v_{1}+4 D_{12}^{0}\right)}{\rho h}}
$$

\section{Results and discussion}

Table 1 presents a comparative study of the dimensionless frequency parameters $\omega_{1}$ for the flexural modes of thin homogeneous isotropic square plates on Winkler foundation. Dimensionless parameters are specified as

$$
\omega_{1}=\frac{\omega b^{2}}{\pi^{2}} \sqrt{\rho h / D^{0}}, \bar{K}=\frac{K a^{4}}{D^{0}}, v_{0}=0.3, \rho=1
$$

Table 1. Comparison of frequency parameters for the flexural modes of thin square plates on homogeneous Winkler elastic foundation $(a / b=1, b / h=100)$

\begin{tabular}{llc}
\hline $\bar{K}$ & Reference & $\omega_{1}$ \\
\hline $10^{2}$ & Leissa [15] & 2.2420 \\
& Zhou et al. [10] & 2.2413 \\
& Present study & 2.2420 \\
$5 \times 10^{2}$ & Leissa [15] & 3.0221 \\
& Zhou et al. [10] & 3.0214 \\
& Present study & 3.0220 \\
\hline
\end{tabular}


Table 2. Variation of frequency parameters of the inhomogeneous orthotropic rectangular plates on inhomogeneous viscoelastic and elastic foundations versus $E_{1} / E_{2}(a / b=0.5, b=1 \mathrm{~m}, a / h=50)$

\begin{tabular}{ccccc}
\hline$E_{1} / E_{2}$ & $\mu_{1}=1$ & $\mu_{1}=2$ & $\mu_{1}=1$ & $\mu_{1}=2$ \\
& $\mu_{2}=0$ & $\mu_{2}=0$ & $\mu_{2}=1$ & $\mu_{2}=1$ \\
\hline$K_{w}^{0}=0$ & & & & \\
10 & 3.084 & 3.777 & 2.953 & 3.616 \\
25 & 2.995 & 3.668 & 2.868 & 3.512 \\
40 & 2.964 & 3.630 & 2.838 & 3.476 \\
55 & 2.947 & 3.609 & 2.821 & 3.455 \\
$K_{w}^{0}=5 \times 10^{6}\left(\mathrm{~N} / \mathrm{m}^{3}\right)$ & & & \\
10 & 3.320 & 3.972 & 3.198 & 3.819 \\
25 & 3.238 & 3.869 & 3.120 & 3.721 \\
40 & 3.209 & 3.833 & 3.093 & 3.687 \\
55 & 3.193 & 3.813 & 3.077 & 3.668 \\
\hline
\end{tabular}

Table 3. Variation of frequency parameters of the inhomogeneous orthotropic rectangular plates on Winkler elastic foundation $a / b \quad(a / h=50, b=1 \mathrm{~m})$

\begin{tabular}{lllll}
\hline$a / b$ & $\mu_{1}=1$ & $\mu_{1}=2$ & $\mu_{1}=1$ & $\mu_{1}=2$ \\
& $\mu_{2}=0$ & $\mu_{2}=0$ & $\mu_{2}=1$ & $\mu_{2}=1$ \\
\hline$K_{w}^{0}=0$ & & & & \\
0.5 & 2.931 & 3.590 & 2.806 & 3.437 \\
1.0 & 3.237 & 3.964 & 3.099 & 3.795 \\
1.5 & 3.957 & 4.847 & 3.789 & 4.640 \\
2.0 & 5.216 & 6.388 & 4.994 & 6.116 \\
$K_{w}^{0}=5 \times 10^{6}\left(\mathrm{~N} / \mathrm{m}^{3}\right)$ & & & \\
0.5 & 3.178 & 3.795 & 3.064 & 3.650 \\
1.0 & 3.674 & 4.328 & 3.553 & 4.174 \\
1.5 & 4.493 & 5.293 & 4.346 & 5.105 \\
2.0 & 5.766 & 6.844 & 5.566 & 6.591 \\
\hline
\end{tabular}

These values are taken from the study of Leissa [15]. As seen, the natural frequencies are in good agreement with the results of Leissa [15] and Zhou et al. [10] for thinner plates.

Variation of the dimensionless frequency parameters for homogeneous and inhomogeneous rectangular plates with and without Winkler elastic foundation against $E_{1} / E_{2}$ ratio for $a / b=0.5, b=$ $1 \mathrm{~m}$ and $a / h=0.5$ are presented in Table 2. The material properties of orthotropic plate are $E_{1} / E_{2}=$ $10,25,40$ and 50, $E_{2}=206.9 G P a, G_{12}=0.5 E_{2}$, $v_{1}=0.25, \rho=1 \mathrm{~kg} / \mathrm{m}^{3}$. As Table 2 shows, the dimensionless frequency parameters for the considered cases are weakly decreased when $E_{1} / E_{2}$ ratio increases.

Since the soil effect is not taken into account, the heterogeneity effect is approximately independent of $E_{1} / E_{2}$ ratio, although the heterogeneity effect is considerable. For example, if the foundation effect is not taken into account, the effect of non-homogeneity is significant $(22.47 \%)$, when the Young's and shear moduli change only in $x$ direction, whereas, this effect is found to be $-4.3 \%$, when the Young moduli change only in $z$ direction and the effect of heterogeneity is $\% 17.3$, as the Young's moduli change in $x$ and $z$ directions together. Here, the negative sign indicates that the value for the inhomogeneous plate is smaller than the homogeneous plate. Considering the foundation effect, significant changes occur in the effects of non-homogeneity on the frequencies. For example, as the Winkler foundation coefficient is taken to be $K_{w}=5 \times 10^{6}$ $\mathrm{N} / \mathrm{m}^{3}$, the effect of non-homogeneity on the frequencies increases by up to $29 \%$, as the Young's and shear moduli change only in $x$ direction; this effect increases from $3.7 \%$ to $4.41 \%$, when the Young and shear moduli vary only in $z$ direction, the effect of non-homogeneity a weak increase from $23.83 \%$ to $24.47 \%$, as the Young and shear moduli vary in $x$ and $z$ directions together, as $E_{1} / E_{2}$ ratio increases from 10 to 55 .

Variations of the dimensionless frequency parameters of homogeneous and inhomogeneous rectangular plates with and without Winkler elastic foundation against $a / b$ ratio for $a / h=50$ and $b=$ $1 \mathrm{~m}$ are presented in Table 3. The material properties of orthotropic plate are $E_{1}^{0}=206.9 \mathrm{GPa}$, $E_{2}^{0}=20.69 \mathrm{GPa}, G_{12}^{0}=6.9 \mathrm{GPa}$ and $\rho^{0}=1950 \mathrm{~kg} / \mathrm{m}^{3}$ Table 3 shows that the values $\omega_{1}$ increase with increasing $a / b$. The effect of the Winkler foundation on the frequency values first increases 
and then decreases, as the $a / b$ increases from 0.5 to 2. For example, the effect of Winkler foundation on the frequencies of homogeneous orthotropic plates chances between $8.43 \%$ and $13.55 \%$. For the inhomogeneous orthotropic plates, the effect of elastic foundation changes in the range of $29.48 \%$ and $33.76 \%$, as the Young's and shear moduli change only in $x$ direction. This effect changes in the range of $4.54 \%$ and $9.76 \%$, when the Young's and shear moduli vary only in the $z$ direction, and the range of the influence of the inhomogeneity is $24.53 \%$ to $29.01 \%$, as the Young and shear moduli vary in the $\mathrm{x}$ and $\mathrm{z}$ directions together, since the ratio $a / b$ increases from 0.5 to 2 .

\section{Conclusions}

The purpose of this study is to investigate the effects of the inhomogeneous and elastic foundation on the frequency of orthotropic plates. The inhomogeneity of orthotropic materials varies linearly with the coordinates of the length and thickness. The basic equation is derived applying the Donnell-Mushtari theory and solved using the Galerkin's method. The effects of inhomogeneity, Winkler elastic foundation and orthotropy on the frequencies are investigated in detail.

\section{References}

[1] Kolchin AS, Favarion EA (1977) Theory of Elasticity of Inhomogeneous Bodies. Chisinau, USSR.

[2] Haciyev VC, Sofiyev AH, Mirzoyev RD (1996) Free vibration of non-homogeneous elastic rectangular plates. Proceedings of Institute of Mathematics and Mechanics, Academy of Sciences of Azerbaijan, IV (XIII), 103-108, Baku (in Russian).

[3] Sofiyev AH, Aksogan O (1999) Dynamic stability of a non-homogeneous orthotropic elastic cylindrical shell under a time dependent external pressure. Technical Journal, Turkish Chamber of Civil Engineers 10:607-612.

[4] Fares ME, Zenkour AM (1999) Buckling and free vibration of non-homogeneous composite crossply laminated plates with various plate theories. Composite Structures 44:279-287.
[5] Sofiyev AH, Omurtag MH, Schnack E (2009) The vibration and stability of orthotropic conical shells with non-homogeneous material properties under a hydrostatic pressure. Journal Sound and Vibration 319(3-5):963-983.

[6] Xia P, Long SY, Cui HX, Li GY (2009) The static and free vibration analysis of a nonhomogeneous moderately thick plate using the meshless local radial point interpolation method. Engineering Analysis with Boundary Elements 33:770-777.

[7] Lal R, Kumar Y (2013) Transverse vibrations of nonhomogeneous rectangular plates with variable thickness. Mechanics of Advanced Materials and Structures 20:264-275.

[8] Gorbunov-Possadov MN, Malikova TA, Solomin VI (1984) Design of Structures on Elastic Foundation. Strojizdat, Moscow, Russia.

[9] Omurtag MH, Kadioğlu F (1998) Free vibration analysis of orthotropic plates resting on Pasternak foundation by mixed finite element formulation. Computers and Structures 67:253-265.

[10] Zhou D, Cheung YK, Lo SH, Au FTK (2004) Three-dimensional vibration analysis of rectangular thick plates on Pasternak foundation. International Journal for Numerical Methods Engineering 59:1313-1334.

[11] Morimoto T, Tanigawa Y (2007) Elastic stability of inhomogeneous thin plates on an elastic foundation. Archives Applied Mechanics 77(9):653-674.

[12] Hsu MH (2010) Vibration analysis of orthotropic rectangular plates on elastic foundations. Composite Structures 92:844-852.

[13] Lal R, R Saini (2015) Buckling and vibration analysis of non-homogeneous rectangular Kirchhoff plates resting on two-parameter foundation. Meccanica 50(4):893-913.

[14] Ma'en SS, Al-Kouz WG (2016) Vibration analysis of non-uniform orthotropic Kirchhoff plates resting on elastic foundation based on nonlocal elasticity theory. International Journal of Mechanical Sciences 114:1-11.

[15] Leissa AW (1973) The free vibration of plates. Journal of Sound Vibration 31:257-293.

[16] Reddy JN (2004) Mechanics of Laminated Composite Plates and Shells: Theory and Analysis. 2nd ed. CRC Press. 